\title{
THE COMPLETENESS OF PROPOSITIONAL RESOLUTION A SIMPLE AND CONSTRUCTIVE PROOF
}

\author{
JEAN GALLIER
}

CIS Department, University of Pennsylvania, Philadelphia, PA 19104, USA

e-mail address: jean@cis.upenn.edu

\begin{abstract}
It is well known that the resolution method (for propositional logic) is complete. However, completeness proofs found in the literature use an argument by contradiction showing that if a set of clauses is unsatisfiable, then it must have a resolution refutation. As a consequence, none of these proofs actually gives an algorithm for producing a resolution refutation from an unsatisfiable set of clauses. In this note, we give a simple and constructive proof of the completeness of propositional resolution which consists of an algorithm together with a proof of its correctness.
\end{abstract}

\section{INTRODUCTION}

The resolution method for (propositional) logic due to J.A. Robinson [4] (1965) is wellknown to be a sound and complete procedure for checking the unsatisfiability of a set of clauses. However, it appears that the completeness proofs that can be found in the literature (for instance, Chang and Lee [1], Lewis and Papadimitriou [3], Robinson [5]) are existence proofs that proceed by contradiction to show that if a set of clauses is unsatisfiable, then it must have a resolution refutation because otherwise a satisfying assignment can be obtained. In particular, none of these proofs yields (directly) an algorithm producing a resolution refutation from an unsatisfiable set of clauses. In that sense, these proofs are nonconstructive. In Gallier [2] (1986), we gave a completeness proof based on an algorithm for converting a Gentzen-like proof (using sequents) into a resolution DAG (see Chapter 4). Such a method is more constructive than the others but, we found later on that it is possible to give a simple and constructive proof of the completeness of propositional resolution which consists of an algorithm together with a proof of its correctness. This algorithm and its correctness are the object of this note.

It should be noted that Judith Underwood gave other constructive proof procedures in her Ph.D. thesis, notably for the intuitionistic propositional calculus [6].

2000 ACM Subject Classification: F.4.1, I.2 .

Key words and phrases: Resolution method, Unsatisfiability, Completeness, Constructive proof.

(C) J. Gallier (c) Creative Commons 


\section{Review of Propositional Resolution}

Recall that a literal, $L$, is either a propositional letter, $P$, or the negation, $\neg P$, of a propositional letter. A clause is a finite set of literals, $\left\{L_{1}, \ldots, L_{k}\right\}$, interpreted as the disjunction $L_{1} \vee \cdots \vee L_{k}$ (when $k=0$, this is the empty clause denoted $\square$ ). A set of clauses, $\Gamma=\left\{C_{1}, \ldots, C_{n}\right\}$, is interpreted as the conjunction $C_{1} \wedge \cdots \wedge C_{n}$. For short, we write $\Gamma=C_{1}, \ldots, C_{n}$.

The resolution method (J.A. Robinson [4]) is a procedure for checking whether a set of clauses, $\Gamma$, is unsatisfiable. The resolution method consists in building a certain kind of labeled DAG whose leaves are labeled with clauses in $\Gamma$ and whose interior nodes are labeled according to the resolution rule. Given two clauses $C=A \cup\{P\}$ and $C^{\prime}=B \cup\{\neg P\}$ (where $P$ is a propositional letter, $P \notin A$ and $\neg P \notin B$ ), the resolvent of $C$ and $C^{\prime}$ is the clause

$$
R=A \cup B
$$

obtained by cancelling out $P$ and $\neg P$. A resolution $D A G$ for $\Gamma$ is a DAG whose leaves are labeled with clauses from $\Gamma$ and such that every interior node $n$ has exactly two predecessors, $n_{1}$ and $n_{2}$ so that $n$ is labeled with the resolvent of the clauses labeling $n_{1}$ and $n_{2}$. In a resolution step involving the nodes, $n_{1}, n_{2}$ and $n$, as above, we say that the two clauses $C$ and $C^{\prime}$ labeling the nodes $n_{1}$ and $n_{2}$ are the parent clauses of the resolvent clause, $R$, labeling the node $n$. In a resolution DAG, $D$, a clause, $C^{\prime}$ is said to be a descendant of a clause, $C$, iff there is a (directed) path from some node labeled with $C$ to a node labeled with $C^{\prime}$. A resolution refutation for $\Gamma$ is a resolution DAG with a single root whose label is the empty clause. (For more details on the resolution method, resolution DAGs, etc., one may consult Gallier [2], Chapter 4, or any of the books cited in Section 1,)

Here is an example of a resolution refutation for the set of clauses

$$
\Gamma=\{\{P, Q\},\{P, \neg Q\},\{\neg P, Q\},\{\neg P, \neg Q\}\}
$$

shown in Figure 1:

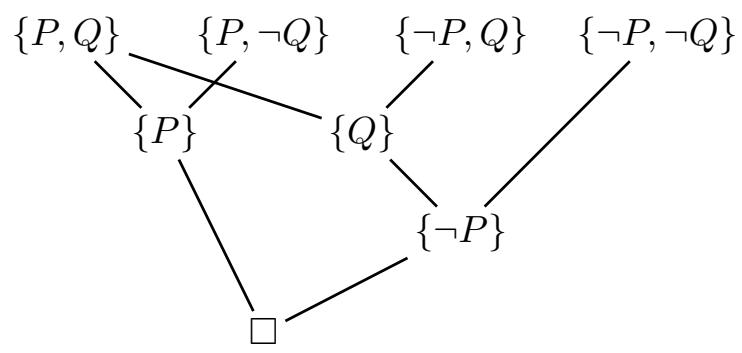

Figure 1: A Resolution Refutation

\section{Completeness of Propositional Resolution: An Algorithm And its CorRectness}

Let $\Gamma$ be a set of clauses. Thus, $\Gamma$ is either the empty clause, $\square$, or it is a conjunction of clauses, $\Gamma=C_{1}, \ldots, C_{n}$. We define the complexity, $c(C)$, of a clause, $C$, as the number of disjunction symbols in $C$; i.e., if $C$ consists of a single literal (i.e., $C=\{L\}$, for some literal, $L$ ), then $c(C)=0$, else if $C=\left\{L_{1}, \ldots, L_{m}\right\}$ (with $m \geq 2$ ) where the $L_{i}$ 's are literals, 
then $c(C)=m-1$ (we also set $c(\square)=0$ ). If $\Gamma$ is a conjunction of clauses, $\Gamma=C_{1}, \ldots, C_{n}$, then we set

$$
c(\Gamma)=c\left(C_{1}\right)+\cdots+c\left(C_{n}\right) .
$$

We now give a recursive algorithm, buildresol, for constructing a resolution DAG from any set of clauses and then prove its correctness, namely, that if the input set of clauses is unsatisfiable, then the output resolution DAG is a resolution refutation. This establishes the completeness of propositional resolution constructively.

Our algorithm makes use of two functions, percolate, and graft.

1. The function percolate $(D, A, L)$

The inputs are: a resolution DAG, $D$, some selected leaf of $D$ labeled with a clause, $A$, and some literal, $L$. This function adds the literal $L$ to the clause $A$ to form the clause $A \cup\{L\}$ and then "percolates" $L$ down to the root of $D$. More precisely, we construct the resolution DAG, $D^{\prime}$, whose underlying unlabeled DAG is identical to $D$, as follows: Since $D$ and $D^{\prime}$ have the same unlabeled DAG we refer to two nodes of $D$ of $D^{\prime}$ as corresponding nodes if they are identical in the underlying unlabeled DAG. Consider any resolution step of $D$. If both parent clauses are not descendants of the premise $A$, then the corresponding resolution step of $D^{\prime}$ is the same. If the parent clauses in $D$ are $C$ and $C^{\prime}$ where $C^{\prime}$ is a descendant of the premise $A$ (resp. $C$ is a descendant of the premise $A$ ) and if $R$ is the resolvent ot $C$ and $C^{\prime}$ in $D$, then the corresponding parent nodes in $D^{\prime}$ are labeled with $C$ and $C^{\prime} \cup\{L\}$ and their resolvent node with $R \cup\{L\}$ (resp. the corresponding parent nodes in $D^{\prime}$ are labeled with $C \cup\{L\}$ and $C^{\prime}$ and their resolvent node wih $R \cup\{L\}$ ). If both parent clauses $C$ and $C^{\prime}$ in $D$ are descendant of the premise $A$, then the corresponding parent nodes in $D^{\prime}$ are labeled with $C \cup\{L\}$ and $C^{\prime} \cup\{L\}$ and their resolvent node with $R \cup\{L\}$.

Observe that if $\Delta \cup\{A\}$ is the set of premises of $D$, then $\Gamma=\Delta \cup\{A \cup\{L\}\}$ is the set of premises of percolate $(D, A, L)$.

For example, if $D$ is the resolution DAG shown in Figure 2 (in fact, a resolution refutation)

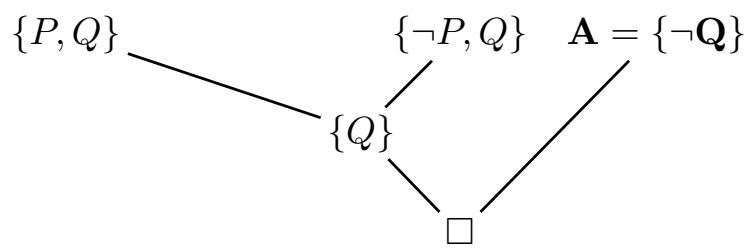

Figure 2: Resolution DAG $D$

then adding $L=\neg P$ to $A=\{\neg Q\}$ in $D$ yields the resolution DAG $D^{\prime}$ produced by percolate $(D, A, L)$ shown in Figure 3 :

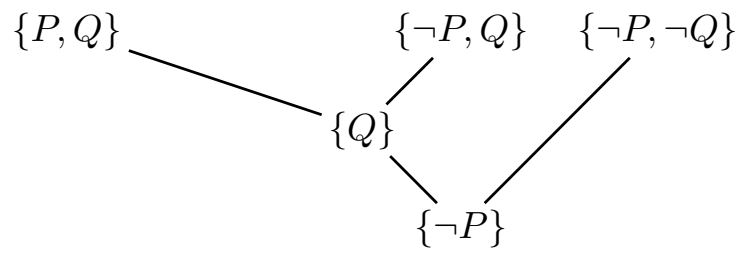

Figure 3: Resolution DAG $D^{\prime}=$ percolate $(D, A, L)$

2. The function $\operatorname{graft}\left(D_{1}, D_{2}\right)$ 
Its inputs are two resolution DAGs, $D_{1}$ and $D_{2}$, where the clause, $C$, labeling the root of $D_{1}$ is identical to one of the premises of $D_{2}$. Then, this function combines $D_{1}$ and $D_{2}$ by connecting the links to the premise labeled $C$ in $D_{2}$ to the root of $D_{1}$, also labeled $C$, obtaining the resolution DAG graft $\left(D_{1}, D_{2}\right)$.

For example, if $D_{1}$ and $D_{2}$ are the resolution refutation DAGs shown in Figure 4 and Figure 5

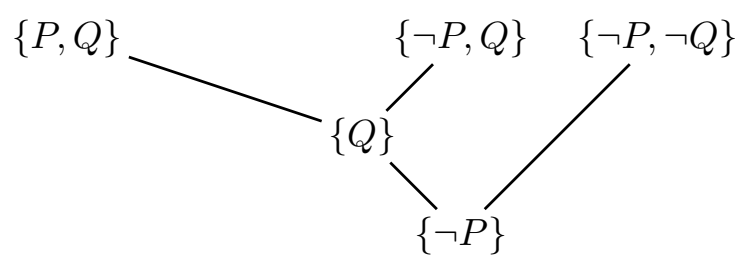

Figure 4: Resolution DAG $D_{1}$

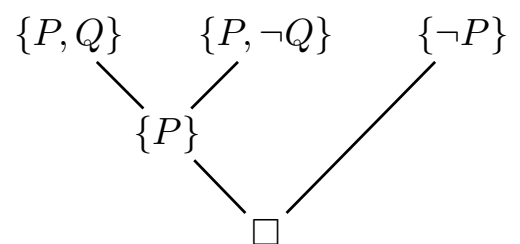

Figure 5: Resolution DAG $D_{2}$

we obtain the resolution DAG in Figure 6

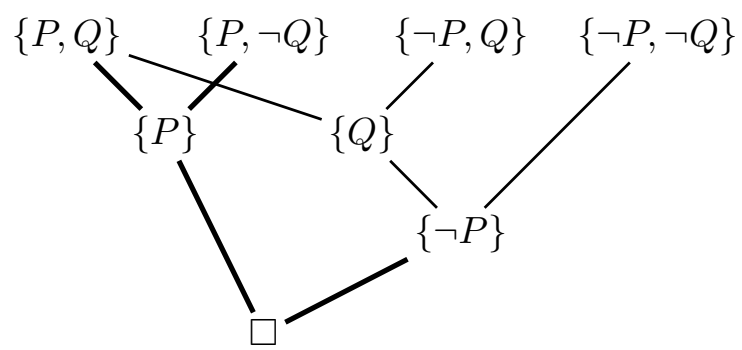

Figure 6: Resolution DAG graft $\left(D_{1}, D_{2}\right)$

where the edges coming from $D_{2}$ are indicated with thicker lines. The algorithm buildresol is shown below.

3. The algorithm buildresol $(\Gamma)$

The input to buildresol is a set of clauses, $\Gamma$.

function buildresol $(\Gamma)$

begin

if all clauses in $\Gamma$ are literals then

if $\Gamma$ contains complementary literals $L$ and $\neg L$,

then return a resolution refutation with leaves $L$ and $\neg L$

else abort

endif 
else select any nonliteral clause, $C$, in $\Gamma$ and select any literal, $L$, in $C$;

let $C=A \cup\{L\}$; let $\Gamma=\Delta \cup\{C\}$;

$D_{1}=\operatorname{buildresol}(\Delta \cup\{A\}) ; D_{2}=\operatorname{buildresol}(\Delta \cup\{L\}) ; D_{1}^{\prime}=\operatorname{percolate}\left(D_{1}, A, L\right)$;

if $D_{1}^{\prime}$ is a resolution DAG

then return $D_{1}^{\prime}$

else $D=\operatorname{graft}\left(D_{1}^{\prime}, D_{2}\right) ; \operatorname{return} D$

endif

endif

end

Finally, we prove the correctness of our recursive algorithm buildresol.

Theorem 3.1. For every conjunction of clauses, $\Gamma$, if $\Gamma$ is unsatisfiable, then the algorithm builresol outputs a resolution refutation for $\Gamma$. Therefore, propositional resolution is complete.

Proof. We prove the correctness of the algorithm buildresol by induction on $c(\Gamma)$. Let $\Gamma=C_{1}, \ldots, C_{n}$. We may assume $\Gamma \neq \square$, since the case $\Gamma=\square$ is trivial. We proceed by induction on $c(\Gamma)$.

If $c(\Gamma)=0$, then every clause, $C_{i}$, contains a single literal and if $\Gamma$ is unsatisfiable, then there must be two complementary clauses, $C_{i}=\{P\}$ and $C_{j}=\{\neg P\}$, in $\Gamma$. Thus, we instantly get a resolution refutation by applying the resolution rule to $\{P\}$ and $\{\neg P\}$.

Otherwise, $c(\Gamma)>0$, so there is some clause in $\Gamma$ that contains at least two literals. Pick any such clause, $C$, and pick any literal, $L$, in $C$. Write $C=A \cup\{L\}$ with $A \neq \square$ and write $\Gamma=\Delta, C$ ( $\Delta$ can't be empty since $\Gamma$ is unsatisfiable). As $\Gamma=\Delta, A \cup\{L\}$ is unsatisfiable, both $\Delta, A$ and $\Delta, L$ must be unsatisfiable. However, observe that

$$
c(\Delta, A)<c(\Gamma) \text { and } c(\Delta, L)<c(\Gamma) .
$$

Therefore, by the induction hypothesis, the algorithm buildresol produces two resolution refutations, $D_{1}$ and $D_{2}$, with sets of premises $\Delta, A$ and $\Delta, L$, respectively. Now, consider the resolution DAG, $D_{1}^{\prime}=$ percolate $\left(D_{1}, A, L\right)$, obtained from $D_{1}$ by adding $L$ to the clause $A$ and letting $L$ percolate down to the root.

Observe that in $D_{1}^{\prime}$, every clause that is a descendant of the premise $A \cup\{L\}$ is of the form $C \cup\{L\}$, where $C$ is the corresponding clause in $D_{1}$. Therefore, the root of the new DAG $D_{1}^{\prime}$ obtained from $D_{1}$ is either labeled $\square$ (this may happen when the other clause in a resolution step involving a descendent of the clause $A$ already contains $L$ ) or $L$. In the first case, $D_{1}^{\prime}$ is already a resolution refutation for $\Gamma$ and we are done. In the second case, we can combine $D_{1}^{\prime}$ and $D_{2}$ using graft $\left(D_{1}^{\prime}, D_{2}\right)$ since the root of $D_{1}^{\prime}$ is also labeled $L$, one of the premises of $D_{2}$. Clearly, we obtain a resolution refutation for $\Gamma$.

As an illustration of our algorithm, consider the set of clauses

$$
\Gamma=\{\{P, Q\},\{P, \neg Q\},\{\neg P, Q\},\{\neg P, \neg Q\}\}
$$

as above and pick $C=\{\neg P, \neg Q\}, L=\neg P$ and $A=\{\neg Q\}$. After the two calls buildresol $(\Delta \cup\{A\})$ and buildresol $(\Delta \cup\{L\})$, we get the resolution refutations $D_{1}$ shown in Figure 7 ; 


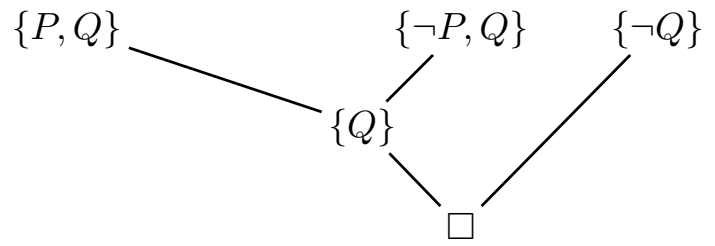

Figure 7: Resolution DAG $D_{1}=\operatorname{buildresol}(\Delta \cup\{A\})$

and $D_{2}$ shown in Figure 8 ;

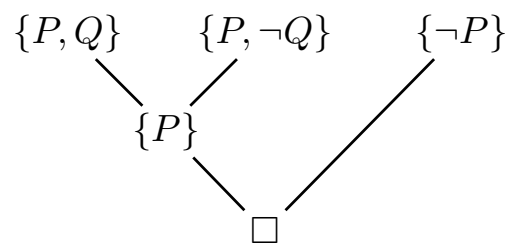

Figure 8: Resolution DAG $D_{2}=\operatorname{buildresol}(\Delta \cup\{L\})$

When we add $L=\neg P$ to $A=\{\neg Q\}$ in $D_{1}$, we get the resolution DAG $D_{1}^{\prime}=$ percolate $\left(D_{1}, A, L\right)$ shown in Figure 9.

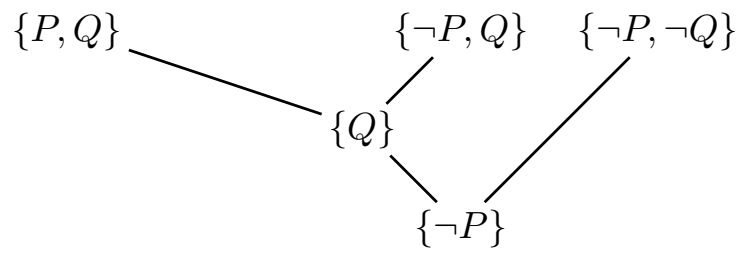

Figure 9: Resolution DAG $D_{1}^{\prime}=\operatorname{percolate}\left(D_{1}, A, L\right)$

Finally, we construct the resolution refutation $D=\operatorname{graft}\left(D_{1}^{\prime}, D_{2}\right)$ shown in Figure 10:

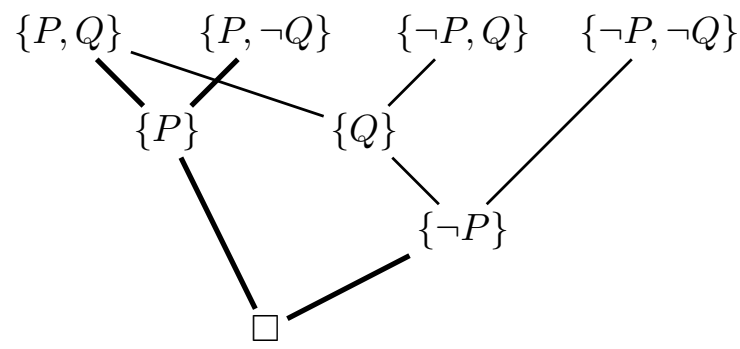

Figure 10: Resolution DAG $D=\operatorname{graft}\left(D_{1}^{\prime}, D_{2}\right)$

where the edges coming from $D_{2}$ are indicated with thicker lines.

Observe that the proof of Theorem 3.1 proves that if $\Gamma$ is unsatisfiable, then our algorithm succeeds no matter which clause containing at least two literals is chosen and no matter which literal is picked in such a clause.

Furthermore, as pointed out by one of the referees, although the proof of completeness is constructive in the sense that it shows an algorithm is correct, it does not explicitly use constructive logic. Nevertheless the logical proof can be recovered from the algorithm and it is constructive. 


\section{ACKNOWLEDGEMENT}

The author wishes to thank Robert Constable and the referees for very helpful comments.

\section{REFERENCES}

[1] Chin-Liang Chang and Richard Char-Tung Lee. Symbolic Logic and Mechanical Theorem Proving. Academic Press, first edition, 1973.

[2] Jean H. Gallier. Logic For Computer Science. Wiley, first edition, 1986.

[3] Harry R. Lewis and Christos H. Papadimitriou. Elements of the Theory of Computation. Prentice-Hall, first edition, 1981.

[4] J.A. Robinson. A machine oriented logic based on the resolution principle. J.ACM, 12(1):23-41, 1965.

[5] J.A. Robinson. Logic: Form and Function. North-Holland, first edition, 1979.

[6] Judith Underwood. The tableau algorithm for intuitionistic propositional calculus as a constructive completeness proof. In Basin D., Fronhofer B., Hahnle R., Posegga J., and Schwind C., editors, Second Workshop on Theorem Proving with Analytic Tableaux and Related Methods, Marseille, France, pages 245-248. Max-Planck-Institut fur Informatik, Saarbrucken, Germany, 1993. 